Макарова Елена Львовна, Новоселова Мария Александровна

\title{
ВОЗМОЖНОСТИ И ОГРАНИЧЕНИЯ ИСПОЛЬЗОВАНИЯ МЕТОДА РЕАЛЬНЫХ ОПЦИОНОВ ПРИ ОЦЕНКЕ ИННОВАЦИОННЫХ ПРОЕКТОВ ${ }^{1}$
}

Целью настоящей работы является исследование возможностей и ограничений использования метода реальных опционов при оченке инновационных проектов. Для достижения поставлениой цели был проведен анализ теоретического, аналитического и методического обеспечения и эмпирического опытта реализации инновационных проектов, на основании результатов которого были определены основные характеристики метода реальных опционов. Основные ограничения использования метода реальньх опционов состоят в необходимости обеспечения получения достоверныхх исходных данныхх, опасности завылиения стоимости убыточного инновационного проекта, пересмотре планов и рисках недостижения целей компании, отсутствии поддерэки в корпоративной культуре компании, отсутствии квалифицированных специалистов и средств поддержки принятия решений по структурированию реальных опционов в инновационных проектах. Для построения управления инновационными проектами с позиций методологии реальных опционов необходимо реализовывать представленный в статье алгоритм определения реальных опционов.

ключевые слова: оценка эффективности, инновачионный проект, реальные опционы.

\section{Elena Makarova, Maria Novoselova \\ OPPORTUNITIES AND LIMITATIONS OF USING THE METHOD \\ OF REAL OPTIONS IN EVALUATION OF INNOVATION PROJECTS}

The purpose of this study is to investigate the possibilities and limitations of using the real option method when evaluating innovative projects. To achieve this goal, an analysis of the theoretical, analytical, empirical and methodological support for the implementation of innovative projects was made, based on the results of which the main characteristics of the real options method were determined. The main limitations of using the real option method are the necessity to ensure the receipt of reliable initial data, the risk of overstating the cost of a loss-making innovative project, the revision of plans and the risks of not achieving the company's goals, lack of a support in the company's corporate culture and lack of qualified specialists, and decision support tools for structuring real options in innovative projects. To build the management of innovative projects from the standpoint of the methodology of real options, it is necessary to implement the algorithm for determining real options presented in the article.

Key words: effectiveness evaluation, innovation project, real options.

Bведение / Introduction. Современные геополитические внешние условия и ограничение доступа к мировым рынкам капитала обусловливают необходимость для отечественного бизнеса привлекать внутренние инвестиционные ресурсы с целью реализации инвестиционных проектов. Внедрение инноваций для многих российских компаний становятся жизненно необходимым для повышения конкурентоспособности на сужающемся отечественном рынке, так как их конкурентные преимущества возникают благодаря постоянным инновациям и гибкости, в связи с чем реанимируется проблематика разработки подходов к управлению инновационными проектами. Это актуализирует вопросы поиска инструментария управления и оценки эффективности инновационных проектов и механизмов повышения эффективности их реализации.

\footnotetext{
Исследование выполнено при финансовой подлержке РФФИ, в рамках научного проекта № 18-010-00793 «Разработка экономико-математического инструментария управления и оценки эффективности инновационных проектов на базе биномиальных моделей»

The reported study was supported by Russian Foundation for Basic Research, project "Development of economic and mathematical tools for
} managing and evaluating the effectiveness of innovative projects based on binomial models", № 18-010-00793 
Одной из причин низкой инновационной активности российских компаний является эндогенная сложность реализации самих инновационных проектов. Эти проекты, в отличие от виолентных секторов производства товаров и услуг повседневного спроса, обычно долгосрочны и дорогостоящи, требуют координации ресурсов в течение нескольких последовательно и параллельно выполняемых этапов. Их отличительными чертами являются: неопределенность результатов; высокие технические и коммерческие риски; сложность оценки влияния стратегического эффекта; высокая стоимость и продолжительность разработки и внедрения. Также оказывают влияние и экзогенные факторы внешней среды: диспропорции российской системы инвестирования инновационной деятельности и макросистемные проблемы финансирования инноваций в России.

Maтериалы и методы / Materials and methods. Методы оценки инвестиционных проектов не вполне адекватно подходят для анализа инновационных проектов, так как имеет место иманнентно связанная со спецификой инновационных проектов неопределенность их финансовых результатов. Концепция использования реальных опционов как философия и методология оценки эффективности инновационных проектов появилась вследствие невозможности традиционных методов инвестиционного анализа непосредственно учитывать стоимость управленческой гибкости и возможность маневра при реализации инновационных проектов более 40 лет назад в работах Ф. Блэка, М. Шоулза и Р. Мертона (1973), С. Майерса (1977), Л. Тригеоргиса (1993), Р. МакГрата, Дж. Розенбергера, А. Дамодарана, которые адаптировали методический аппарат оценки финансовых опционов для отбора инвестиционных проектов [1-4].

Часто используемый на практике и распространенный метод дисконтирования денежных притоков и оттоков проекта DCF (Discounted Cash Flow Method) предполагает оценку эффективности проекта в относительно стационарной среде с определенной вероятностью и основан на прогнозе основного варианта развития реализации проекта. Аналитики в целях избежания неопределенности в момент проектного анализа могут рассматривать еще два сценария: пессимистический и оптимистический. В результате обычно берется за основу «усредненный вариант» развития проекта и «усредненный риск». Возможный риск проекта кумулятивно учитывается в значении ставки дисконтирования.

На практике высокий риск инновационной деятельности требует применения повышенной премии за риск инвестирования, что приводит к снижению приведенных дисконтированных значений доходов и расходов проекта и, следовательно, не соответствует реальности. Кроме того, в инновационных проектах часто встречается нестандартный ряд денежных потоков. Поэтому механизм дисконтирования потоков проекта адекватно отражает ситуацию только при безрисковой ставке с учетом риска в самих значениях денежных потоков, без учета премии за риск.

Таким образом, необходимость учета «управленческой гибкости» и различных сценариев реализации проекта предопределяют возможность и необходимость использования реальных опционов при проведении оценки эффективности инновационных проектов.

В реальных условиях высокодинамичной недетерминированной стохастичной внешней среды реализации инновационного проекта диапазон потенциально возможных управленческих решений и разнообразие вариантов различных стратегий реализации инновационных проектов увеличиваются, и стратегическая стоимость опционов имеет существенную ценность для менеджмента компании при наличии изменяющихся эндогенных и экзогенных факторов, что и обусловливает использование метода реальных опционов при реализации инновационных проектов.

Технологии конструирования и встраивания реальных опционов в области инвестиционного анализа позволяют учесть высокие риски и особенности вероятности результата различных этапов инновационного процесса при оценке эффективности и управлении реализацией инновационных проектов. 
Структуризация реальных опционов в инновационных проектах представляет деятельность компании в виде совокупности инвестиционных проектов и дает возможность сделать множественные решения от первоначальной идеи инновации до ее завершения независимыми друг от друга, позволяя структурировать инновационный проект как многоэтапное решение с соответствующими исходами. Реальные опционы дают возможность увеличивать гибкость в достижении намеченных целей и осуществлять дополнительное пошаговое инвестирование и позволяют при менеджменте проекта не руководствоваться усредненными прогнозами, а определять альтернативные пути развития проекта в ситуациях волатильности и неопределенности при возникновении дополнительной информации или изменении условий его реализации. Применение метода реальных опционов позволяет, например, в рамках реализации крупномасштабного проекта, состоящего из нескольких этапов, изменять возможность, структуру или сроки реализации каждого этапа при радикальном увеличении волатильности проекта. Стратегический анализ реальных опционов развития проекта является основой принятия управленческих решений на долгосрочную перспективу в условиях риска и неопределенности.

Результаты и об̈суждение / Results and discussion. На основании анализа специфики, возможностей и ограничений применения метода реальных опционов к оценке инновационных проектов можно определить основные рекомендации по его применению в практике инвестиционного менеджмента.

Сферы применения метода реальных опционов обширны. Во всех сферах, где имеется неопределенность, инновационный проект можно структурировать на основе метода реальных опционов.

Востребовано применение реальных опционов в ситуациях принятия решений в условиях неопределенности на рынках:

- с высокой волатильностью либо на несформировавшихся рынках;

- при продвижении новых продуктов и проектах с высокими маркетинговыми расходами;

- на монопольных рынках или в случае, когда результат или продукт инновационного проекта является образующим или единственным на рынке, поэтому действия менеджмента проекта очень сильно влияют на конъюнктуру такого рынка;

- $\quad$ в сырьевых секторах и commodity сегментах из-за высоких рисков колебания спроса и предложения и гиперзависимости от часто меняющейся конъюнктуры рынка и географических, политических, экономических, социальных, психологических и других факторов.

Инновационные проекты, реализуемые в отраслях, «чувствительных» к соблюдению стандартов и законодательства подвержены дополнительным неопределенностям специализированного законодательства, международных норм, ограничений, санкций и соглашений:

- проекты в отраслях химии, металлургии, нефтепереработки, транспорта связаны с экологическими стандартами, нормами и ограничениями (киотский протокол, экологические налоги и т. д.);

- инфраструктурные проекты, как правило, масштабны, технически сложно реализуемы и рискованны, подвержены всему спектру неопределенностей: рискам регуляторного характера, недостаточной проработке законодательства, возможным изменениям законодательства или внешним ограничениям [5].

Метод реальных опционов эффективно применяется:

- в оценке стоимости инвестиций в нематериальные активы, (исследования, патентные отношения и т. п.), находящиеся в процессе разработки, когда коммерческую жизнеспособность доказать не представляется возможным;

- проектах, предусматривающих сделки с интеллектуальной собственностью;

- проектах, использующих схемы проектного финансирования; 
- высокотехнологичных, наукоемких, ресурсных отраслях;

- проектах финансового сотрудничества государства и бизнеса, механизмах государственно-частного партнерства;

- биотехнологиях и фармацевтике;

- $\quad$ новых технологиях в телекоммуникациях и электронике;

- химической, нефтяной промышленности;

- сельском хозяйстве;

- проектно-конструкторских работах при высоких рисках сложных инвестиционных проектов;

- геологоразведочных проектах;

- строительстве недвижимости и девелоперских проектах.

Возможности использования метода реальных опционов возникают:

- при отрицательном или близком к нулю значении показателя NPV проекта;

- наличии высокой степени неопределенности результатов инновационного проекта;

- зависимости финансового результата проекта от принимаемых менеджментом решений;

- наличии сложноструктурируемых многостадийных инвестиционных проектов;

- $\quad$ наличии у менеджмента проекта желания и возможности принимать гибкие решения на большом количестве контрольных точек проекта при возникновении дополнительной информации или изменении условий реализации проекта [6];

- наличии особой важности инновационного проекта для менеджмента с целью более точного определения фундаментальных факторов и рисков проекта и хеджировании его провала и неэффективности реализации и обоснования альтернативных вариантов развития проекта [7].

- $\quad$ в start-up бизнесах и проектах венчурного финансирования;

- $\quad$ в проектах, реализуемых на рынках и в условиях с высокими параметрами неопределенности: в кризисных ситуациях или в сложном финансовом положении.

Основные ограничения, требующие осторожности в использования метода реальных опционов, состоят:

- в необходимости обеспечения получения достоверных исходных данных для расчетов;

- необходимости устранении двойного счета опционов;

- в том, что не все имеющиеся возможности представляют собой реальные опционы;

- опасности завышения стоимости убыточного инновационного проекта [8];

- принятии за возможный опцион на выбор времени временных коммерческих преимуществ за счет первенства на рынке и стратегии «снятия сливок» [9];

- частом пересмотре планов и расфокусировке целей стратегии компании вследствие реализации управленческой гибкости, предоставляемой опционами [10];

- отсутствии ликвидного рынка реальных опционов и непрерывной стохастической динамики цены актива и бесконечной повторяемости, именно на которые и рассчитана применяемая для оценки формула Блэка - Шоулза;

- сложности использования бинарного дерева Кокса - Росса - Рубинштейна в ситуациях роста количества временных периодов, что обусловлено механизмом построения модели, а также невозможностью использовать модель при наличии более двух источников неопределенности [11];

- применении единой ставки дисконтирования (усредненной или индивидуальной) во всех расчетных периодах проекта для каждого звена бинарного дерева Кокса - Росса Рубинштейна, так как ставка дисконтирования состоит из разных видов риска, которые меняются разнонаправленно в различных периодах, что искажает итоговые результаты оценки [12]; 
- применении на практике количественно не оцененной аналитиками формулы расчета стоимости реального опциона как суммы традиционного и опционного чистого дисконтированного дохода [12];

- использовании сложного математического аппарата и отсутствии сформированной методики определения и построения портфеля инновационных проектов на базе метода реальных опционов проекта;

- снижении мотивации менеджмента и сотрудников проекта на недопущение провала и прекращения проекта и сосредоточении максимума возможных усилий на достижении результата из-за возможности отказа от реализации проекта и необходимости компенсации падения мотивации соответствующими управленческими решениями [13];

- отсутствии поддержки в корпоративной культуре компании, неумении или нежелании менеджеров и сотрудников компании быть гибкими и мобильными;

- отсутствии квалифицированных специалистов, накопленного российского опыта и адаптированной к специфике российской инновационной деятельности методологии и методик для различных сфер реализации инновационных проектов и программных продуктов для поддержки принятия решений по структурированию инновационных проектов с использованием реальных опционов.

Для построения управления инновационными проектами с позиций методологии реальных опционов можно представить следующий алгоритм определения наличия реальных опционов:

- определение источников неопределенности как в деятельности компании в целом, так и при реализации инновационного проекта в частности;

- анализ экзогенных факторов, оказывающих влияние на проект и компанию для определения наиболее существенных источников неопределенности, способных повлиять на их функционирование;

- отбор источников неопределенности, уровень вероятности и неопределенности которых можно оценить количественно;

- проведение количественной оценки уровня вероятности и неопределенности каждого выявленного источника неопределенности;

- генерирование и отбор управленческих решений, нацеленных на формирование реального опциона;

- определение сфер, этапов, стадий, бизнес-процессов инновационного проекта, которые подвержены влиянию определенных ранее источников неопределенности;

- $\quad$ анализ сфер, этапов, стадий, бизнес-процессов инновационного проекта, определение и отбор управленческих решений, на которые выявленные источники неопределенности влияют с эффектом повышения стоимости опциона;

- $\quad$ анализ эффектов и коммерческой эффективности принятия подобных управленческих решений;

- составление перечня управленческих решений и его анализ с точки зрения значимости влияния на стоимость реализуемого инновационного проекта;

- анализ вероятности получения дополнительной стоимости инновационного проекта [14].

Заключение / Conclusion. Необходимо отметить, что в большинстве случаев анализа реализуемых инновационных проектов использование только метода DCF является достаточным. Использование метода реальных опционов необоснованно, если инновационный проект имеет высокую степень достоверности и высокую чистую приведенную стоимость. При наличии высокого NPV достаточно иметь информацию, что проект содержит некие опционы, которые также имеют ценность. Использование более трудоемкого метода реальных опционов обоснованно, если его применение улучшает точность показателей и ценность самого проекта. 
При принятии управленческих решений по реализации инновационного проекта менеджерам необходимо понимать, что деятельность компании может включать реальные опционы, необходимо учитывать это и в максимальной степени использовать данные преимущества. Однако нельзя отождествлять реальный опцион с выбором и необходимо дифференцировать его с ситуациями выбора времени, сроков, рисков и других параметров осуществления инвестиций. При наличии сомнений в ценности опциона необходимо применять количественные методы оценки реальных опционов. Также при всех достоинствах реальных опционов как метода оценки эффективности инновационных проектов он не универсален, и уместность его применения должна рассматриваться для отдельно взятый проект. Определение стоимости опционов на базе модели дерева решений предполагает только два возможных исхода от реализации проекта: начало и расширение или закрытие проекта, однако этот метод не применим к анализу проектов по созданию стратегического потенциала компании, при использовании дополнительных методов анализа, например имитационного моделирования, возможным становится получить комбинированный точный прогноз положительных и отрицательных последствий применения метода реальных опционов.

Встраивание анализа реальных опционов для определения стратегических возможностей и условной стоимости в традиционную схему менеджмента инновационного проекта позволяет объединить потенциалы инвестиционного и стратегического анализа и позволяет получить релевантный аналитический инструмент для анализа экономических процессов и разработать эффективные решения при реализации инновационного проекта в экономике риска и неопределенности.

\section{ЛИТЕРАТУРА И ИНТЕРНЕТ-РЕСУРСЫ}

1. Black, F., Scholes M. The Pricing of Options and Corporate Liabilities // Journal of Political Economy. 1973. № 81. Pp. 637-659.

2. Myers S. Determinants of corporate borrowing // Journal of Financial Economics. 1977. № 5. Pp. 147-175.

3. Trigeorgis L. Real Options and Interactions with Financial Flexibility // Financial Management. 1993. № 22 (3). Pp. 202-224.

4. Демодоран А. Инвестиционная оценка: Инструменты и техника оценки любых активов / пер. с англ. 5-е изд. М.: Альпина Бизнес Букс, 2008. 1340 с.

5. Просолупова К. С. Анализ неопределенности: особенности современного финансового анализа // Аудит и финансовый анализ. 2012. № 3. С. 172-177.

6. Трифонов Ю. В., Кошелев Е. В., Купцов А. В. Российская модель метода реальных опционов // Вестник Нижегородского университета им. Н. И. Лобачевского. 2012. № 2 (1). С. 238-243.

7. Трифонов Ю. В., Яшин С. Н., Кошелев Е. В. Применение реальных опционов для инвестирования инноваций в условиях ограниченности информации // Финансы и кредит. 2011. № 30(462). С. 2-9.

8. Рош Дж. Стоимость компании: От желаемого к действительному. Минск: Гревцов Паблишер, 2008. 352 с.

9. Инвестиционный менеджмент: учебник / Л. Д. Капранова, С. Н. Малофеев, Т. Н. Седаш; под ред. Н. И. Лахметкиной. М.: КноРус, 2014. 262 с.

10. Крюков С. В. Оценка инновационных проектов в «пространстве реальных опционов» // Terra Economicus. 2011. T. 9. № 2. Ч. 3. С. 58-62.

11. Алексеева Н. А., Бякова А. В. Концептуальные основы управления реализацией исследовательских проектов по утилизации попутного нефтяного газа в РФ с использованием методики реальных опционов // Глобальный научный потенциал. 2013. № 1(22). С. 46-54.

12. Баев Л. А., Егорова О. В., Правдина Н. В. К вопросу о применении теории реальных опционов в оценке и управлении инвестиционными проектами // Вестник Уральского института экономики, управления и права. 2010. № 20. С. 59-68.

13. Leslie K. J, Michaels M. P. The real power of real options // The McKinsey Quarterly. 2000. № 3. 


\section{REFERENCES AND INTERNET RESOURCES}

1. Black F., Scholes M. The Pricing of Options and Corporate Liabilities // Journal of Political Economy. 1973. № 81. Pp. 637-659.

2. Myers S. Determinants of corporate borrowing // Journal of Financial Economics. 1977. № 5. Pp. 147-175.

3. Trigeorgis L. Real Options and Interactions with Financial Flexibility // Financial Management. 1993. № 22 (3). Pp. 202-224.

4. Demodoran A. Investitsionnaya otsenka: Instrumenty i tekhnika otsenki lyubykh aktivov (Investment appraisal: Tools and techniques for valuing any assets) / per. s angl. 5-e izd. M. : Al'pina Biznes Buks. 2008. 1340 p.

5. Prosolupova K. S. Analiz neopredelennosti: osobennosti sovremennogo finansovogo analiza (Uncertainty analysis: features of modern financial analysis) // Audit i finansovyy analiz. 2012. № 3. pp. 172-177

6. Trifonov YU. V., Koshelev Ye. V., Kuptsov A. V. Rossiyskaya model' metoda real'nykh optsionov (Russian model of the method of real options)// Vestnik Nizhegorodskogo universiteta im. N.I. Lobachevskogo. 2012. № 2 (1).pp. 238-243.

7. Trifonov YU. V., YAshin S. N., Koshelev E. V. Primenenie real'nyh opcionov dlya investirovaniya innovacij $\mathrm{v}$ usloviyah ogranichennosti informacii (Application of real options for investment of innovations in conditions of limited information) // Finansy i kredit. 2011. № 30(462). S. 2-9.

8. Rosh J. Stoimost' kompanii: Ot zhelayemogo k deystvitel'nomu (Company value: From desired to real). Minsk: Grevtsov Pablisher, 2008. 352 s.

9. Investitsionnyy menedzhment: uchebnik (Investment management: textbook) / L. D. Kapranova, S. N. Malofeyev, T. N. Sedash; pod red. N. I. Lakhmetkinoi. M.: KnoRus, 2014. $262 \mathrm{~s}$.

10. Kryukov S. V. Otsenka innovatsionnykh proyektov v «prostranstve real'nykh optsionov» (Evaluation of innovative projects in the «real options space») // Terra Economicus. 2011. T. 9. № 2. CH. 3. S. 58-62.

11. Alekseyeva N. A., Byakova A. V. Kontseptual'nyye osnovy upravleniya realizatsiyey issledovatel'skikh proyektov po utilizatsii poputnogo neftyanogo gaza v RF s ispol'zovaniyem metodiki real'nykh optsionov (Conceptual framework for managing the implementation of research projects for the utilization of associated petroleum gas in the Russian Federation using the real options technique) // Global'nyy nauchnyy potentsial. 2013. № 1(22). S. 46-54.

12. Bayev L. A., Yegorova O. V., Pravdina N. V. K voprosu o primenenii teorii real'nykh optsionov v otsenke i upravlenii investitsionnymi proyektami (On the issue of the application of the theory of real options in the evaluation and management of investment projects) // Vestnik Ural'skogo instituta ekonomiki, upravleniya i prava. 2010. № 20. S. 59-68.

13. Leslie K. J, Michaels M. P. The real power of real options // The McKinsey Quarterly. 2000. № 3.

\section{СВЕДЕНИЯ ОБ АВТОРАХ}

Макарова Елена Львовна, кандидат педагогических наук, доцент кафедры менеджмента и инновационных технологий, Институт управления в экономических, экологических и социальных системах Южного федерального университета. E-mail: elmakarova $@$ sfedu.ru

Новоселова Мария Александровна, аспирант кафедры экономической теории и национальной экономики, Саратовский государственный университет. E-mail: mariya_nov@mail.ru

\section{INFORMATION ABOUT AUTHORS}

Makarova Elena L., Candidate of pedagogical sciences, Associate professor of Management and innovative technologies department, Institute of Management in Economic, Environmental and Social Systems, Southern Federal University. E-mail: elmakarova@sfedu.ru

Novosepova Maria A., Aspirant of economic theory and national economics department, Saratov State University. E-mail: mariya_nov@mail.ru 